\title{
Primary cytomegalovirus infection with accompanying Pneumocystis jiroveci pneumonia in a patient with large-vessel vasculitis
}

\author{
M. Vetter $\cdot$ M. Battegay $\cdot$ M. Trendelenburg
}

Received: 13 January 2010/Accepted: 17 March 2010/Published online: 15 April 2010

(c) Urban \& Vogel 2010

\begin{abstract}
A 70 year-old female patient presented with fever, nausea and dyspnea. She had been receiving immunosuppressive therapy with methotrexate and prednisone for large-vessel vasculitis. The patient was shown to have coexistent Pneumocystis jiroveci pneumonia and primary cytomegalovirus (CMV) infection with presumed CMV pneumonitis and colitis. To our knowledge, this is the first case report on the occurrence of combined primary cytomegalovirus and Pneumocystis jiroveci infection in a patient with vasculitis. It illustrates the importance of being aware of the possibility of combined opportunistic infections in patients with rheumatologic diseases.
\end{abstract}

Keywords Large-vessel vasculitis - Immunosuppressive therapy $\cdot$ Pneumocystis jiroveci pneumonia .

Cytomegalovirus infection

\section{Introduction}

Opportunistic infections frequently occur in immunocompromised patients such as HIV-infected patients, transplant recipients receiving immunosuppressive therapy and patients with neoplasm or rheumatologic diseases. In patients with rheumatologic diseases, opportunistic infections can largely be attributed to steroid use and low

M. Vetter $(\bowtie) \cdot$ M. Trendelenburg

Department of Internal Medicine, University Hospital Basel, Petersgraben 4, 4031 Basel, Switzerland

e-mail: vetterm@uhbs.ch

M. Battegay

Division of Infectious Diseases and Hospital Epidemiology, University Hospital Basel, Petersgraben 4, 4031 Basel, Switzerland lymphocyte counts [1-3]. Complications due to these infections are most frequent in patients with systemic lupus erythematosus (SLE) and to a lesser extent in rheumatoid arthritis (RA) [4]. They also present a major risk to patients with systemic vasculitis [5].

Cytomegalovirus (CMV) is a DNA virus belonging to the herpes viridae family. It remains latent within the body after the initial infection and can be reactivated when the immune system is impaired. Between about 40 and $90 \%$ of the population in central Europe and the US carry cytomegalovirus [6, 7]. CMV disease is frequently reported in immunocompromised patients [8]. Common symptoms are nausea, fever, sweating and abnormal liver function [9]. CMV diseases reported in the literature are usually due to reactivation of CMV in immunocompromised patients and transplant recipients $[10,11]$. Over $90 \%$ of elderly persons have antibodies against CMV indicating previous primary infection [6]. Thus, primary CMV infection rarely occurs in the elderly.

CMV disease may be accompanied by co-infections. This may partly be due to its immunosuppressive effect [12]. Combined cytomegalovirus and Pneumocystis jiroveci infection has been described in the literature and may be fatal [13]. It generally affects young patients with HIV-AIDS and reactivation of the cytomegalovirus [14].

Here we present a 70 year-old patient with the unique combination of primary cytomegalovirus infection, Pneumocystis jiroveci pneumonia and vasculitis. The unusual occurrence of primary cytomegalovirus infection in this age group emphasizes the importance of close monitoring of immunosuppressed patients in all age groups. Elderly immunosuppressed patients have a greater likelihood of contracting more than one opportunistic infection, thereby increasing the risk of a fatal outcome. 


\section{Case report}

The 70 year-old female patient was admitted to our hospital with elevated body temperature (up to $38.3^{\circ} \mathrm{C}$ ) and progressive nausea. She had been receiving oral steroids (prednisone $30 \mathrm{mg} /$ day) and methotrexate once a week for 5 weeks for unclassified ANCA-negative large-vessel vasculitis, which had been diagnosed 1 year earlier. The patient had a history of arterial hypertension, glaucoma, chronic bronchiectasia with lobe resection of the left lung in 1977, partial thyroidectomy due to nodule in 1997 , breast cancer with surgery, chemotherapy and radiation in 1997, tuberculosis in 1947 and partial bowel resection due to diverticulitis.

On admission, the patient complained of fatigue, nausea, vomiting, listlessness and mild dyspnea. These symptoms had started 3 weeks earlier, directly after a methotrexate injection. In the week preceding admission, she had developed temperatures of up to $38.3^{\circ} \mathrm{C}$, usually in the morning. Two days before admission, the nausea had increased and she had started to throw up.

No focus of infection could be found in the physical examination. Urine and blood cultures were negative. Pathologic laboratory findings are summarized Table 1. CT scan of chest and abdomen showed no signs of inflammation. Chest X-ray showed also no interstitial pneumonitis. Serologic tests and bronchoalveolar lavage (BAL) were performed. Serology showed the pattern of primary cytomegalovirus infection with positive CMV IgG antibodies and IgM antibodies of intermediate avidity. Active cytomegalovirus infection was confirmed by positive antigenemia with a viral load of two million copies (PCR). A retrospective analysis of frozen serum samples taken
1 year earlier was negative for $\operatorname{IgG}$ and $\operatorname{IgM}$ anti-CMV antibodies.

Cytomegalovirus was also isolated from BAL fluid with 5.18 million copies in the PCR. Bronchoscopy of the left inferior lobe demonstrates a severe inflammation of the bronchial system (Fig. 1). BAL fluid also showed an unexpected positive cytology for Pneumocystis jiroveci (immunofluorescence in situ hybridization).

Methotrexate was discontinued and steroids were reduced to $20 \mathrm{mg}$ prednisone per day. Treatment was initiated with intravenous pentamidine (due to known trimethoprim/sulfamethoxazole allergy) for 8 days and ganciclovir for 10 days. This therapy resulted in complete recovery within 10 days and the patient was discharged 17 days after admission. Drug prophylaxis was initiated for CMV and PCP infection. Follow-up visits were uneventful 1 year after discharge.

\section{Discussion}

Our case report demonstrates the unique occurrence of combined Pneumocystis jiroveci pneumonia and primary cytomegalovirus infection in an elderly patient with largevessel vasculitis.

Symptomatic CMV infections in immunocompetent patients usually occur in younger age groups and are rare in the elderly [15]. The diagnosis of primary CMV infection in this 70 year-old patient was based on serologic findings showing seroconversion and a high titer of CMV IgM antibodies of intermediate avidity. The patient also had positive antigenemia and a high viral load as determined by PCR. It is most likely that the patient's gastrointestinal

Table 1 Pathological findings in the laboratory

\begin{tabular}{lllll}
\hline Pathologic laboratory findings & On admission & On discharge & Maximal values & Normal values \\
\hline C-reactive protein (mg/l) & 31.0 & 8.9 & 60.8 & $0-10.0$ \\
White blood cell count (G/l) & 2.41 & 14.1 & 17.0 & $3.5-10.0$ \\
Lymphocytes (G/l) & 3.36 & 4.59 & 5.25 & $0.9-3.3$ \\
CD4+ counts (cells/mcl) & - & - & 1198 & $700-1100$ \\
ASAT (U/l) & 42 & 59 & 336 & $11-36$ \\
ALAT (U/l) & 63 & 126 & 454 & $10-37$ \\
GGT (U/l) & 75 & 361 & 519 & $8-49$ \\
Bilirubin mcmol/l & 12 & 11 & 13 & $5-18$ \\
CMV-IgG (S/CO) & 2.5 & - & - & $<0.90$ \\
CMV-IgG Avidity (Index) & 0.201 & - & - & $<0.30$ \\
CMV-IgM (S/CO) & 5.4 & - & - & $<0.90$ \\
CMV PCR (copies/ml) in BAL & $5.18 \times 10^{6}$ & - & - & ND \\
CMV PCR (copies/ml) in blood & $2.00 \times 10^{6}$ & &
\end{tabular}

$B A L$ bronchoalveolar lavage fluid 


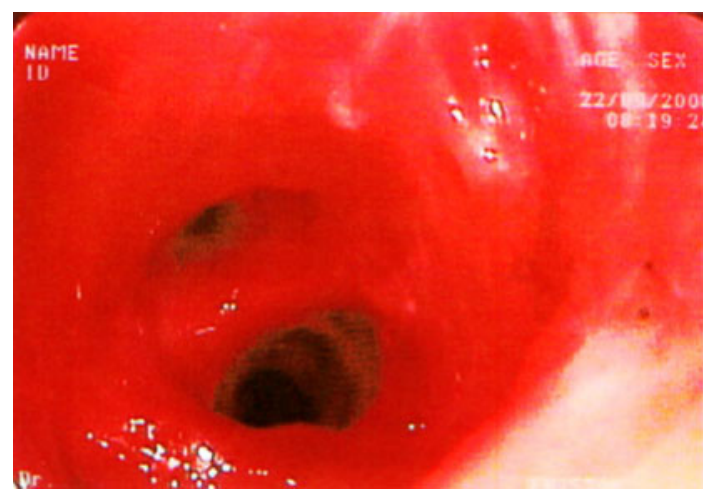

Fig. 1 Bronchoscopy of the left inferior lobe demonstrated a severe inflammation of the bronchial system. Bronchoscope contact caused bleeding

symptoms were caused by the primary CMV infection, but no colonoscopy was performed to confirm CMV colitis. CMV was also isolated from BAL fluid suggesting CMV pneumonia. However, a positive BAL finding for CMV is common in patients with multiple opportunistic pulmonary infections, including Pneumocystis jiroveci infection, and cannot generally be equated to CMV pneumonia [15]. A study of asymptomatic HIV-infected subjects showed that BAL cultures were positive for CMV in nine out of 19 subjects irrespective of their pulmonary symptoms [16].

CMV infection coexisting with PCP is of clinical relevance, however. In a Danish study it could be shown that the mortality of patients receiving steroids for Pneumocystis jiroveci infection doubled when there was concomitant CMV infection [17]. Niolles et al. [18] suggested that CMV infection was responsible for fatal outcomes in patients developing opportunistic fungal and bacterial infections after hematopoietic stem cell transplantation.

The unique feature in our case was the occurrence of combined primary CMV infection and Pneumocystis jiroveci pneumonia in an elderly patient with vasculitis. Rheumatologic diseases including vasculitis themselves can cause immunosuppression. In addition, the patient received an immunosuppressive regimen of steroids and methotrexate. Steroid treatment has been shown to be a risk factor for opportunistic infections in patients with rheumatologic diseases [1-3, 19]. While immunosuppressive therapy is important for controlling the underlying disease, it should not be initiated without considering the risk of infection. In order to prevent cytomegalovirus and pneumocystis infection, chemoprophylaxis is warranted in patients receiving immunosuppressive therapy and patients with previous disorders such as pulmonary disease [20]. Furthermore, physicians should be aware of the possibility of combined opportunistic infections.
Conflict of interest statement None.

\section{References}

1. Hedderwick SA, Bonilla HF, Bradley SF, Kauffman CA. Opportunistic infections in patients with temporal arteritis treated with corticosteroids. J Am Geriatr Soc. 1997;45:334-7.

2. Ognibene FP, Shelhamer JH, Hoffman GS, Kerr GS, Reda D, Fauci AS, et al. Pneumocystis carinii pneumonia: a major complication of immunosuppressive therapy in patients with Wegener's granulomatosis. Am J Respir Crit Care Med. 1995;151:795-9.

3. Sendi P, Wolf A, Graber P, Zimmerli W. Multiple opportunistic infections after high-dose steroid therapy for giant cell arteritis in a patient previously treated with a purine analog. Scand J Infect Dis. 2006;38:922-4.

4. Staples PJ, Gerding DN, Decker JL, Gordon RS Jr. Incidence of infection in systemic lupus erythematosus. Arthritis Rheum. 1974;17:1-10.

5. Sneller MC. Evaluation, treatment, and prophylaxis of infections complicating systemic vasculitis. Curr Opin Rheumatol. 1998;10:38-44.

6. Staras SA, Dollard SC, Radford KW, Flanders WD, Pass RF, Cannon MJ. Seroprevalence of cytomegalovirus infection in the United States, 1988-1994. Clin Infect Dis. 2006;43:1143-51.

7. Lübeck PR, Doerr HW, Rabenau HF. Epidemiology of human cytomegalovirus (HCMV) in an urban region of Germany: what has changed? Med Microbiol Immunol. 2010;199:53-60. (Epub 2009 Dec 1).

8. Merigan TC. Immunosuppression and herpesviruses. In: Nahmias AJ, Dowdle WR, Schinazi RF editors, The human herpesviruses: an interdisciplinary perspective. New York: Elsevier, 1981:30922.

9. Wreghitt TG, Teare EL, Sule O, Devi R, Rice P. Cytomegalovirus infection in immunocompetent patients. Clin Infect Dis. 2003:37:1603-6.

10. Griffiths PD. Current management of cytomegalovirus disease. J Med Virol. 1993;1:106-11.

11. Sutherland S, Bracken P, Wreghitt TG, O'Grady J, Calne RY, Williams R. Donated organ as a source of cytomegalovirus in orthotopic liver transplantation. J Med Virol. 1992;37:170-3.

12. Boeckh M, Nichols WG. Immunosuppressive effects of betaherpesviruses. Herpes. 2003;10:12-6.

13. Samuel R, Bettiker RL, Suh B. AIDS related opportunistic infections, going but not gone. Arch Pharm Res. 2002;25:215-28.

14. Graham SM. HIV-related pulmonary disorders: practice issues. Ann Trop Paediatr. 2007;27:243-52.

15. Wreghitt TG, Teare EL, Sule O, Devi R, Rice P. Cytomegalovirus infection in immunocompetent patients. Clin Infect Dis. 2003;37:1603-6.

16. Mann M, Shelhamer JH, Masur H, Gill VJ, Travis W, Solomon $\mathrm{D}$, et al. Lack of clinical utility of bronchoalveolar lavage cultures for cytomegalovirus in HIV infection. Am J Respir Crit Care Med. 1997;155:1723-8.

17. Jensen AM, Lundgren JD, Benfield T, Nielsen TL, Vestbo J. Does cytomegalovirus predict a poor prognosis in Pneumocystis carinii pneumonia treated with corticosteroids? A note for caution. Chest. 1995;108:411-4.

18. Nichols WG, Corey L, Gooley T, Davis C, Boeckh M. High risk of death due to bacterial and fungal infection among cytomegalovirus (CMV)-seronegative recipients of stem cell transplants from seropositive donors: evidence for indirect effects of primary 
CMV infection. J Infect Dis. 2002;185:273-82 (Epub 2002 Jan 17).

19. Greenberg SB. Infections in the immunocompromised rheumatologic patient. Crit Care Clin. 2002;18:931-56.
20. Mounzer KC, DiNubile MJ. Prophylactic use of antibiotics and vaccines in patients with rheumatologic disorders. Rheum Dis Clin North Am. 1997;23:259-75. 